\title{
Pain and fatigue in adult patients with rheumatoid arthritis: Association with body awareness, demographic, disease-related, emotional and psychosocial factors*
}

\author{
Helena Lööf ${ }^{1,2 \#, ~ U n n-B r i t t ~ J o h a n s s o n ~}{ }^{1,2}$, Elisabet Welin Henriksson ${ }^{3}$, Staffan Lindblad ${ }^{4}$, \\ Fredrik Saboonchi ${ }^{2,5,6,7}$ \\ ${ }^{1}$ Sophiahemmet University, Stockholm, Sweden \\ ${ }^{2}$ Karolinska Institutet, Department of Clinical Sciences, Danderyd Hospital, Division of Medicine, Stockholm, Sweden \\ ${ }^{3}$ Karolinska Institutet, Division of Nursing, Department of Neurobiology and Rheumatology Unit, Karolinska Hospital, Stockholm, \\ Sweden \\ ${ }^{4}$ Karolinska Institutet, Department of Learning Informatics, Management and Ethics, Stockholm, Sweden \\ ${ }^{5}$ Karolinska Institutet, Department of Neuroscience, Division of Insurance Medicine, Stockholm, Sweden \\ ${ }^{6}$ University of Stockholm, Stress Research Institute, Stockholm, Sweden \\ ${ }^{7}$ Red Cross University College, Stockholm, Sweden \\ Email: " helena.loof@sophiahemmethogskola.se
}

Received 31 January 2013; revised 1 April 2013; accepted 15 May 2013

Copyright (C) 2013 Helena Lööf et al. This is an open access article distributed under the Creative Commons Attribution License, which permits unrestricted use, distribution, and reproduction in any medium, provided the original work is properly cited.

\begin{abstract}
Background: Patients and clinicians report pain and fatigue as key outcome measures in rheumatoid arthritis. Fatigue and pain are a major concern to patients. Aim: The objective of this study was to examine fatigue and pain in adult patients with rheumatoid arthritis (RA) and to investigate the association between pain and fatigue with body awareness, demographic, disease-related, emotional and psychosocial factors. Method: Data were collected from a sample of patients with RA $(n=120)$ recruited from a Rheumatology clinic in a large university hospital in Stockholm, Sweden. Eligible for inclusion were patients between 20 - 80 years of age and with a confirmed diagnosis of RA. Fatigue was measured using the Multidimensional Assessment of Fatigue (MAF) scale, while the Visual Analogue Scale (VAS) was used to assess components of pain. A multiple stepwise regression analysis was performed to evaluate factors related to fatigue and pain. In the first step a univariate analysis of variance (ANOVA) was used for all relevant independent factors. In the next step backwards stepwise regression was applied. Result: Fatigue was significantly associated with the Disease
\end{abstract}

\footnotetext{
"This study was supported by research grants from Sophiahemmet University and the Sophiahemmet Foundation, the MSD (Merck Sharp \& Dohme, AB Sweden) and the FRS, Association of Rheumatology Nurses in Sweden.

"Corresponding author.
}

Activity Score 28-joints (DAS 28) $(p=0.049)$, the Body Awareness Questionnaire (BAQ) $(p=0.006)$, the Positive Affect (PA) scale $(p=0.008)$ and no smoking $(p=0.021)$. Pain was significantly associated with the EuroQol EQ-5D $(p=0.008)$ and the DAS 28 $(p=0.001)$. The adjusted R-square was $28.6 \%$ for fatigue and $50.0 \%$ for pain. Conclusion: This study clearly demonstrates that fatigue and pain in patients with RA appear to be associated with disease-related factors. Furthermore, fatigue was related to body awareness and emotional factors, and pain was related to health related quality of life.

Keywords: Pain; Fatigue; Emotional; Psychosocial; Rheumatoid Arthritis

\section{INTRODUCTION}

Clinically significant fatigue is common in patients with rheumatoid arthritis (RA) [1]. Patients with RA continue to report moderate to severe pain [2], both fatigue and pain are a major concern to patients [3,4]. Patients and clinicians report pain and fatigue as key outcome measures in RA [5,6]. Furthermore, fatigue is recommended [7] as a patient-centered outcome measure in RA. Fatigue predicts quality of life (QoL) [8] and pain impacts QoL [9]. The psychological well-being of the individual living with RA is significantly affected by the fundamental life changes and the complexity of the disease process $[10,11]$. 
Fatigue in RA is associated with the severity of pain, disease activity, functional status [12], comorbidity, disability, multiple social roles and low social support [13-15]. Scientific support is available indicating that sex, disease duration, pain and functional status all influence fatigue in RA $[15,16]$. In a study [17], the authors report that pain severity, role functioning, depressive mood, self-efficacy on fatigue, helplessness, worrying and nonrestorative sleep are factors most strongly associated with fatigue level. Variables described in a review [18] related to fatigue are illness related aspects, physical functioning, cognitive/emotional functioning and social environmental aspects. Some possible consequences of fatigue are psychological distress, reduced QoL and work ability.

The tendency to focus attention on bodily sensations and internal stimuli, i.e. body awareness [19] has been associated with an increase in both somatic and emotional distress [20]. A biopsychosocial approach has been suggested to be applicable in the study of chronic diseases in general [21] and RA in particular [22]. In such an approach psychological factors are considered not merely affected by the course of the disease but also important for the patients health and well-being [23]. RA impacts on daily life, especially when performing physical activities. In addition, it has a pronounced effect on mood and social life [24]. Negative emotionality and stress are among major psychological factors that have been associated with RA [25]. The impact of negative emotion in the context can be observed as negative influences on health behavior [26]. Furthermore, emotions have a crucial role in how people adjust to having RA, and in the context of chronic pain in general [27,28]. Negatively toned self-focused bodily attention has been linked to less effective decision-making strategies and worse adherence in patients with other chronic diseases [29].

Because many symptoms of RA (i.e. pain and fatigue) are generally evocative of negative emotional responses, elevated body awareness may be associated with worse perceived health. Both emotional and attention related processes in the individual occur in a social context. The objective of this study was to examine fatigue and pain in adult patients with RA and to investigate the association between pain and fatigue with body awareness, demographic, disease-related, emotional and psychosocial factors.

\section{METHOD}

\subsection{Design}

This was a cross-sectional questionnaire survey with a descriptive design.

\subsection{Population}

The study population consisted of a sample of 120 patients with RA attending a physician consultation at the
Rheumatology clinic, Karolinska University Hospital, Sweden. Eligible for inclusion were patients aged 20 - 80 years with a confirmed diagnosis of RA according to the American College of Rheumatology (ACR) criteria for RA [30]. The patients should have been diagnosed with RA for at least a period of six months, speak and understand the Swedish language as well as read and comprehend the study instructions. Exclusion criteria were another serious disease (for example, Parkinson or Multiple sclerosis) that could significantly affect the outcome of the study.

\subsection{Instrument}

The Multidimensional Assessment of Fatigue (MAF) measures four dimensions of fatigue: severity, distress, degree of interference in activities of daily living, and timing. Respondents were asked to reflect on any patterns of fatigue that have occurred over the past week [16]. The Swedish version of MAF has been tested in patients with rheumatic disease (systemic sclerosis) [31].

The VAS was used to assess components of pain occurring over the past week. The patients were asked one question about pain: "How much pain did you have during the past week because of your rheumatic disease?" [32].

Demographic data was collected and included sex (female/male), age (year), smoking (yes or no), educational status (compulsory school, upper secondary school, higher education and other education) and marital status (single or common-law). Physical activity per week was categorized into yes $=\geq 7$ days of physical activity per week and no $=<7$ days of physical activity per week. Working status was categorized into yes $=$ working and no $=$ not working and retired and sick leave.

Disease activity was evaluated using the Disease Activity Score 28-joints (DAS 28). A score, below 3.2 indicates low disease activity and a score above 5.1 indicates high disease activity. DAS 28 is based on erythrocyte sedimentation rate $(\mathrm{ESR}, \mathrm{mm} / \mathrm{h})$, number of swollen $(n=$ $28)$ and tender $(n=28)$ joints and on the patients perceived general health (VAS, $0-100 \mathrm{~mm}$ ) [33].

The EuroQol, EQ-5D was used to assess components of health related QoL (HRQoL). EQ-5D includes measures related to mobility, hygiene, daily activities, pain/ discomfort and anxiety/depression. The questionnaire measures preference-based HRQoL on a 0 to 1 scale, where 0 indicates the worst possible health and 1 full health [34]. It has been suggested to be a valid measure for HRQoL in RA patients [35]

The Body Awareness Questionnaire (BAQ) was used to assess components of body awareness. Higher scores indicate a higher degree of body awareness. The value of Cronbach's alpha has been reported in previous studies to be between 0.80 and $0.88[19,36]$. The Swedish validated version of the BAQ scale was used. In the Swedish version of BAQ Cronbach's alpha was 0.86 [37]. 
The Emotion Regulation Questionnaire (ERQ) was developed to measure the dispositional use of two specific strategies related to emotion control: reappraisal and suppression. In a previous study on these two emotion regulation processes the value of Cronbach's alpha was 0.79 for reappraisal and 0.73 for suppression [38].

The Perceived Stress Scale 4 (PSS4) was employed to assess components of perceived stress. The scale ranges from 0 - 16, with higher scores indicate of a higher degree of perceived stress. Cronbach's alpha for the Perceived Stress Scale was 0.72 [39]. In our study a Swedish version of PSS4 were included and the Cronbach's alpha was 0.82 [40].

The Positive and Negative Affect Scale (PANAS) was applied to assess components of mood. PANAS includes items relating to a positive affect (PA) domain, and to a negative affect domain (NA) domain. A higher score on the PA domain indicates greater PA, or the extent to which the individual feels enthusiastic, active and alert. A higher score on the NA domain represents a greater negative affect or the extent to which the individual feels aversive mood states and general distress. This measure has been validated in a previous study Cronbach's alpha was 0.86 to 0.90 for the PA domain and 0.84 to 0.87 for the NA domain [41]. In a Swedish study the Cronbach's alpha was 0.86 for PA and 0.85 for NA [42].

The Interview Schedule for Social Interaction (ISSI) was used to assess components of social interaction and support. The ISSI consisting of two scales: one describing the availability of deep emotional relationships and attachments, and the other describing the availability of more peripheral contacts of social networks and integration. In previous work Cronbach's alpha was 0.77 for AVSI scale and 0.80 for AVAT scale [43].

\subsection{Data Collection Procedure}

A rheumatologist gave verbal and written information about the study to the patients. The information about the study, and question about participation, were given to the patients after consultation with their physician. After the consultation, the patients were informed that they would receive a letter within a week containing written information about the study (together with several questionnaires and a prepaid envelope). The patients were also informed that the completed questionnaires should be returned within two weeks from date of receipt. No reminder was sent to the patients.

\subsection{Statistics}

Descriptive statistics presented as mean, standard deviation (SD) median, range and percentage were used toassess demographic characteristics of the patients.
To evaluate factors related to fatigue and pain multiple stepwise linear regression analyses were performed. The analyses were performed in two separate models, both performed in two steps. In the first step a univariate ANOVA was conducted on all relevant independent factors. Based on the univariate analyses of variance (ANOVA) all factors with a $p$-value $<0.2$ were entered into the second step. In the second step a backwards stepwise regression was performed with the model selection based on the Akaike information criterion.

When the final model was obtained, the model assumptions were evaluated based on the residual diagnosis. The internal consistency (Cronbach's alpha) for the different measures in our study: 0.86 (BAQ), 0.76 (ERQ-Reappraisal), 0.68 (ERQ-Suppression), 0.90 (PA), 0.89 (NA), 0.76 (AVSI), 0.40 (AVAT) and 0.74 (PSS).

The statistical analyses have been performed in $\mathrm{R}$ version 2.14.1 (R Foundation for Statistical Computing, Vienna, Austria) and IBM SPSS Statistics 20 for Windows (IBM SPSS, NY, USA).

\subsection{Ethical Consideration}

Ethical approval was obtained from the ethics committee of the Regional Ethical Review Board (Dnr. 2010/734-32 and 2009/1795-31/3). All participants gave written informed consent. The anonymity and confidentiality of the participants were guaranteed.

\section{RESULTS}

\subsection{Background Characteristics}

In all, 120 (response rate 78\%) patients with RA participated in the study; $27(22.5 \%)$ aged $<45$ years, 51 (42.5\%) aged $46-65$ years and $42(35 \%)$ aged $>65$ years. The majority of the patients were female (86\%). Demographic data are shown in (Table 1).

The mean GFI score (MAF total score) was $24.48 \pm$ 10.18 and PAIN $31.58 \pm 24.16$ for pain. Descriptive statistics of variables included in the univariate analysis are presented in (Table 2).

\subsection{Univariate Analysis}

The univariate analysis identified 7 of 17 independent factors with a $p$-value of $<0.2$ (no smoking, no physical activity, DAS 28, BAQ, PA, NA and PSS4) in relation to fatigue. For pain 3 of 17 were identified as significant (EQ-5D, DAS 28, PSS4). The $p$-values from the univariate analysis for fatigue and pain are listed in (Table 3).

\subsection{Multiple Regression Analysis}

The results from the stepwise multiple regression analy- 
Table 1. Demographic characteristics.

\begin{tabular}{|c|c|c|}
\hline & & Total \\
\hline & & $(n=120)$ \\
\hline Sex & $n(\%)$ & \\
\hline Male & & $17(14.17)$ \\
\hline Female & & $103(85.83)$ \\
\hline Age & $n(\%)$ & \\
\hline$<45$ years & & $27(22.50)$ \\
\hline $46-65$ years & & $51(42.50)$ \\
\hline$>65$ years & & $42(35.00)$ \\
\hline Smoking & $n(\%)$ & \\
\hline Yes & & $15(12.61)$ \\
\hline No & & $104(87.39)$ \\
\hline Marital status & $n(\%)$ & \\
\hline Single & & $27(22.50)$ \\
\hline Common-law & & $93(77.50)$ \\
\hline Working status & $n(\%)$ & \\
\hline Yes & & $54(45.76)$ \\
\hline No & & $39(33.05)$ \\
\hline Sick leave & & $25(21.19)$ \\
\hline Educational status & $n(\%)$ & \\
\hline Compulsory school & & $32(27)$ \\
\hline Upper secondary school & & $31(26)$ \\
\hline $\begin{array}{l}\text { Higher education } \\
\text { Other education }\end{array}$ & & $\begin{array}{l}44(37) \\
12(10)\end{array}$ \\
\hline Physical activity & $n(\%)$ & \\
\hline Yes & & $31(25.83)$ \\
\hline
\end{tabular}

Table 2. Descriptive statistics of variables.

\begin{tabular}{ccccc}
\hline & Mean \pm SD & Median & Range & Possible scores \\
\hline MAF $(n=119)$ & $24.48 \pm 10.18$ & 25.61 & 1 to 45 & $1-50$ \\
PAIN $(n=110)$ & $31.58 \pm 24.16$ & 30 & 0 to 95 & $0-100$ \\
BAQ $(n=116)$ & $70.00 \pm 19.46$ & 71.5 & 18 to 111 & $18-126$ \\
EQ 5D $(n=96)$ & $0.78 \pm 0.21$ & 0.81 & -0.1 to 1 & $0-1$ \\
DAS 28 $(n=117)$ & $3.17 \pm 1.25$ & 2.96 & 0.68 to 6.99 & $0-10$ \\
ERQ-Reappraisal $(n=115)$ & $25.77 \pm 6.45$ & 26 & 6 to 41 & $4-28$ \\
ERQ-Expressive Suppression $(n=116)$ & $11.84 \pm 4.88$ & 12 & 4 to 24 & $10-50$ \\
PA $(n=112)$ & $31.11 \pm 7.29$ & 31 & 10 to 49 & $10-50$ \\
NA $(n=112)$ & $14.37 \pm 6.13$ & 12 & 10 to 50 & $0-6$ \\
AVSI $(n=120)$ & $3.17 \pm 1.96$ & 3 & 0 to 6 & $0-6$ \\
AVAT $(n=120)$ & $4.68 \pm 1.36$ & 5 & 0 to 6 & $0-16$ \\
PSS $(n=120)$ & $9.25 \pm 2.81$ & 9 & 4 to 16 & 42 \\
\hline
\end{tabular}


Table 3. $p$-values in the univariate analysis of fatigue and pain.

\begin{tabular}{ccc}
\hline & Fatigue & Pain \\
\hline Independent variables & $p$-value & $p$-value \\
\hline Sex & 0.376 & 0.695 \\
Age & 0.791 & 0.308 \\
Smoking & 0.104 & 0.948 \\
Marital status & 0.280 & 0.712 \\
Educational status & 0.747 & 0.051 \\
Physical activity & 0.146 & 0.843 \\
EQ 5D & 0.002 & $<0.001$ \\
DAS 28 & 0.002 & $<0.001$ \\
BAQ & 0.056 & 0.493 \\
ERQ-Reappraisal & 0.164 & 0.522 \\
ERQ-Expressive Suppression & 0.807 & 0.972 \\
PA & 0.001 & 0.248 \\
NA & 0.071 & 0.009 \\
AVSI & 0.061 & 0.868 \\
AVAT & 0.033 & 0.721 \\
PSS & $<0.001$ & 0.001 \\
PAIN & 0.015 & - \\
MAF & - & 0.015 \\
\hline & &
\end{tabular}

sis for fatigue shows that fatigue was significantly associated with no smoking $(p=0.021)$, DAS $28(p=0.049)$, $\operatorname{BAQ}(p=0.006)$ and for PA $(p=0.008)$ (Table 4).

The results from the stepwise multiple regression analysis for pain shows that pain was significantly associated with the EQ-5D $(p=0.008)$ and DAS $28(p=0.001)$. The final models for fatigue and pain were considered acceptable (Tables 4 and 5).

Adjusted R-square was $28.6 \%$ for fatigue and $50.0 \%$ for pain.

\section{DISCUSSION}

Our study clearly demonstrates that fatigue in RA is associated with increased disease activity, increased body awareness, and decreased PA. Previous studies have confirmed that disease activity and functional status influence RA $[15,16]$. Psychological factors may play a crucial role in fatigue. For instance, [17] found that severity and depressive mood are factors strongly associated with fatigue level.

The tendency to focus attention on bodily sensations and internal stimuli (i.e. body awareness) [19] has been associated with increased somatic and emotional distress [20]. In the present study fatigue was found associated with increased body awareness. Negatively toned selffocused bodily attention has been linked to less effective decision making strategies and worse adherence [29].

The concept of body awareness, as well as its association to fatigue is therefore deserving of further research. Previous study [37] describes that body awareness may
Table 4. Stepwise regression to identify predictors of fatigue.

\begin{tabular}{cccc}
\hline & Estimate $^{*}$ & $95 \% \mathrm{CI}$ & $p$-value \\
\hline (Intercept) & 21.07 & $(3.86-38.28)$ & 0.017 \\
Smoking (No) & -7.19 & $(-13.25-1.12)$ & 0.021 \\
Physical activity (No) & 3.95 & $(-0.51-8.42)$ & 0.082 \\
DAS 28 & 1.65 & $(0.01-3.29)$ & 0.049 \\
BAQ & 0.16 & $(0.05-0.27)$ & 0.006 \\
PA & -0.43 & $(-0.75-0.12)$ & 0.008 \\
NA & -0.27 & $(-0.60-0.07)$ & 0.120 \\
PSS & 0.78 & $(-0.03-1.58)$ & 0.058 \\
\hline
\end{tabular}

Adjusted R-square $=28.6 \%$; ${ }^{*}$ Estimated $=$ regression coefficient.

Table 5. Stepwise regression to identify predictors of pain.

\begin{tabular}{cccc}
\hline & Estimate $^{*}$ & $95 \%$ CI & $p$-value \\
\hline (Intercept) & 20.36 & $(-14.71-55.43)$ & 0.252 \\
EQ 5D & -34.98 & $(-60.51-9.46)$ & 0.008 \\
DAS 28 & 9.32 & $(5.40-13.25)$ & $<0.001$ \\
PSS & 1.11 & $(-0.34-2.56)$ & 0.131 \\
\hline
\end{tabular}

Adjusted R-square $=50.0 \%$; ${ }^{*}$ Estimated $=$ regression coefficient.

be useful in the management of chronic disease and can be addressed in nursing.

The PA scale is a generic instrument to measure the emotional state of an individual. In the present study decreased PA in patients with RA was associated with fatigue. Previous studies have shown that PA facilitates approach behavior [44] and continued action [45] i.e. individuals engage more with their environment and are more willing to take part in different activities. A person with PA feels more enthusiastic, active and alert. Low PA is manifested in decreased arousal, energy and activity, as well as the absence of positive feelings (e.g., sadness, lethargy and boredom) [38]. Negative emotionality and stress are among some of the major psychological factors that have been adversely linked to RA [25]. The impact of negative emotion in this context may be viewed as either through negative influence on health behavior or through a neuroendocrine influence on immune function and health [26]. Moreover, emotions are thought to play a crucial role in the adjustment of people with RA, and in the context of chronic pain in general [21,27].

Pain is a central outcome measure in rheumatoid arthritis [2] and patients themselves have suggested that assessment and management of pain should be prioritized [11]. A study [9] showed that patients with RA, who consider their disease to be "somewhat to completely controlled", continue to report moderate to severe pain. It is therefore important to investigate all potential factors that contribute to pain. In our study pain was as- 
sociated with decreased HRQoL and increased disease activity. The EQ-5D captures five health dimensions of which the area pain/discomfort is one of these dimensions. Pain/discomfort is also included in the DAS 28. The adjusted R-square value for pain ( $50 \%$ of the model) was fairly high in our study, suggesting that the model is of moderately to high predictive value.

In our study group $54.5 \%$ were not working or were on sick leave at the time of the study. A study [46] has shown that RA can affect QoL as well as the ability to do paid or unpaid work.

Furthermore, [47] described the importance of recording socioeconomic status in clinical trials because patients with lower socioeconomic status are more likely to experience higher disease activity, lower physical function, and poorer emotional aspects of mental health, lower QoL, and greater pain. Low level of pain, high levels of physical activity, and good lower extremity function at baseline predicted good general health perception [48]. Our study noted that low levels of pain in RA patients still had some influence on perceived health.

A biopsychosocial approach has been considered appropriate to study chronic diseases in general [21] and RA in particular [22]. According to such an approach, psychological factors are not merely affected by the course of the disease, but are also important for the patients general health and well-being [23]. To emphasize a health-oriented and salutogenic theoretical perspective the individuals health-relevant pattern of goals and expectations, social needs and resources, as well as emotions, attention, and activities needs to be addressed. In a study [18] the findings underlined the importance of targeting psychological factors to enhance HRQoL issues in the clinical management of RA patients.

The findings in our study confirm that there is an association between negative PA and fatigue, and that pain was associated with decreased HRQoL and increased disease activity. To apply the biopsychosocial approach to clinical practice, it is of importance that the clinician elict the patients history in the context of life circumstances. The clinician should also determine which aspect of biological, psychological, and social domains that is most important to understanding and promoting the patients health and well-being [49,50].

\subsection{Method Discussion and Limitations}

The present study has limitations. One limitation is that we did not include biological markers of RA. A second limitations concerns using a generic instrument to measure fatigue, which means we could have missed dimensions important to patients with RA. Furthermore, we have no information about the non-respondents (22\%) though the response rate of $78 \%$ can be considered fairly high for this kind of study.
There are a number of problems, in inferring changes and trends over time, using a cross-sectional study [51]. This study found several factors significantly associated with fatigue, the adjusted R-square value (28.6 of the model) was moderate, suggesting that the model is of limited predictive value. In further research it is of importance to include other predictors as quality of sleep and several psychological factors $[17,18]$ which could increase the predictive value.

The authors [18] describe that it is a need of prospective longitudinal studies to find out more about the multicausal pathways of fatigue in RA. In addition, it would also be interesting to use other methods to get a deeper understanding of pain, fatigue and body awareness in adult patients with rheumatoid arthritis. In our experience this is the first study investigating the association between fatigue, pain and body awareness.

In the present study a sample from one geographical region was used. However, the sample was demographically comparable to the sample in a population study carried out in southern Sweden [52]. Still, these results need to be interpreted with caution because of the fairly small sample size $(n=120)$.

\subsection{Conclusion}

In conclusion, this study shows that fatigue and pain in patients with RA appear to be associated with disease-related factors. Fatigue was also related to body awareness and emotional factors, and pain was related to health related quality of life. This relation requires further research.

\subsection{Practice Implications}

The tendency to focus attention on bodily sensations and internal stimuli (i.e. body awareness) has been associated with increased somatic and emotional distress. In the present study fatigue was found associated with increased body awareness. Negatively toned self-focused bodily attention has been linked to less effective decision-making strategies and worse adherence. In future research it is of importance to highlight and address the concept of body awareness.

\section{ACKNOWLEDGEMENTS}

The authors wish to thank Inga Lodin, Birgitta Nordmark, Anders Harju, and Sofia Ernestam at Karolinska University Hospital, Solna and Huddinge, Sweden, for help with the administration of this study. The authors are also grateful to Marcus Thuresson Statisticon AB for assistance with the statistical analysis.

\section{REFERENCES}

[1] Wolfe, F., Hawley, D.J. and Wilson, K. (1996) The 
prevalence and meaning of fatigue in rheumatic disease. Journal of Rheumatology, 23, 1407-1417.

[2] Taylor, P., Manger, B., Alvaro-Gracia, J., Johnstone, R., Gomez-Reino, J., Eberhardt, E., Wolfe, F., Schwartzman, S., Furfaro, N. and Kavanaugh, A. (2010) A patient perceptions concerning pain management in the treatment of rheumatoid arthritis. The Journal of International Medical Research, 8, 1213-1224. doi: $10.1177 / 147323001003800402$

[3] Carr, A., Hewlett, S. and Huges, R. (2003) Rheumatology outcomes: The patient's perspective. Journal of Rheumatology, 30, 880-883.

[4] Heiberg, T., Finset, A., Uhlig, T. and Kvien, T.K. (2005) Seven year changes in health status and priorities for improvement of health in patients with rheumatoid arthritis. Annuals Rheumatic Diseases, 64, 191-195. doi:10.1136/ard.2004.022699

[5] Felson, D.T., Andersson, J.J. and Boers, M. (1993) The American College of Rheumatology preliminary core set of disease activity measures for rheumatoid arthritis clinical trials. Arthritis \& Rheumatism, 36, 729-740. doi:10.1002/art.1780360601

[6] Minnock, P., FitzGerald, O. and Bresnihan, B. (2003) Women with established rheumatoid arthritis perceive pain as the predominant impairment of health status. Rheumatology, 41, 995-1000.

doi:10.1093/rheumatology $/$ keg281

[7] Kirwan, J.R., Minnock, P., Adebajo, A., et al. (2006) Patient's perspective: Fatigue as a recommended patient centered outcome measure in rheumatoid arthritis. Journal of Rheumatology, 34, 1174-1777.

[8] Rupp, I., Boshuizen, H.C., Jacobi, C.E., et al. (2004) Impact of fatigue on health-related quality of life in rheumatoid arthritis. Arthritis \& Rheumatism, 51, 578-585. doi:10.1002/art.20539

[9] Read, E., Mc Eachern, C. and Mitchell, T. (2001) Psychological wellbeing of patients with rheumatoid arthritis. British Journal of Nursing, 10, 1385-1391.

[10] Davis, R.M., Wagner, E.G. and Groves, T. (2000) Advances in managing chronic illness. British Medical Journal, 320, 525-526. doi:10.1136/bmj.320.7234.525

[11] Husted, J., Gladman, D., Farewell, V. and Cook, R. (2001) Health related quality of life of patients with psoriatic arthritis: A comparison with patients with rheumatoid and arthritis. Arthritis Care Research, 45, 151-158. doi:10.1002/1529-0131(200104)45:2<151::AID-ANR168 $\geq 3.0 . \mathrm{CO} ; 2-\mathrm{T}$

[12] Garip, Y., Eser, F., Aktenkin, L. and Bodur, H. (2011) Fatigue in rheumatoid arthritis: Association with severity of pain. Disease activity and functional status. Acta Reumatologica Portuguesa, 36, 364-369.

[13] Croby, L.J. (1991) Factors which contribute to fatigue associated with rheumatoid arthritis. Journal of Advanced Nursing, 16, 974-981. doi:10.1111/j.1365-2648.1991.tb01803.x

[14] Fifield, J., Tennen, H., Reisine, S. and McQuillan, J. (1998) Depression and the long-term risk of pain, fatigue, and disability in patients with rheumatoid arthritis. Ar- thritis \& Rheumatism, 41, 1851-1857. doi:10.1002/1529-0131(199810)41:10<1851::AID-ART1 8>3.0.CO;2-I

[15] Huyser, B.A., Parker, J.C., Thoreson, R., Smarr, K.L., Johnson, J.C. and Hoffman, R. (1998) Predictors of subjective fatigue among individuals with rheumatoid arthritis. Arthritis \& Rheumatism, 41, 2230-2237. doi:10.1002/1529-0131(199812)41:12<2230::AID-ART1 9>3.0.CO;2-D

[16] Belza, B.L., Henke, C.J., Yelin, E.H., Epstein, W.V. and Gilliss, C.L. (1993) Correlates of fatigue in older adults with rheumatoid arthritis. Nursing research, 42, 93-99. doi:10.1097/00006199-199303000-00006

[17] Van Hoogmoed, D., Fransen, J.J., Bleiienberg, G., et al. (2010) Physical and psychosocial correlates of severe fatigue in rheumatoid arthritis. Rheumatology, 49, 1294 1302. doi:10.1093/rheumatology/keq043

[18] Nikolaus, S., Bode, C., Taal, E. and van de Laar, M. (2012) Which factors are related to fatigue in rheumatoid arthritis? Annals of the Rheumatic Diseases. The EULAR Journal, 71, 740-741.

[19] Shields, S.A., Mallroy, M.E. and Simon, A. (1989) The body awareness questionnaire: Reliability and validity. Journal of Personality Assessment, 53, 802-815. doi:10.1207/s15327752jpa5304_16

[20] Ingram, R.E. (1990) Self-focused attention in clinical disorders: Review and conceptual model. Psychological Bullentin, 107, 156-176. doi:10.1037/0033-2909.107.2.156

[21] Hamilton, N.A. and Malcarne, V.L. (2004) Cognition, emotion, and chronic illness. Cognitive Therapy and Research, 28, 555-557. doi:10.1023/B:COTR.0000045564.11322.53

[22] Zautra, A.J. (2003) Comment on "Stress-vulnerabiliy factors as long-term predictors of disease activity in early rheumatoid arthritis". Journal of Psychosomatics Research, 55, 303-304. doi:10.1016/S0022-3999(03)00035-7

[23] Keefe, F.J., Smith, S.J., Buffington, A.L., Gibson, J., Studts, J.L. and Caldwell, D.S. (2002) Recent advances and future directions in the biopsychosocial assessment and treatment of arthritis. Journal of Consulting and Clinical Psychology, 70, 640-655. doi:10.1037/0022-006X.70.3.640

[24] Nyman, S.C. and Lutzen, K. (1999) Caring needs of patients with rheumatoid arthritis. Nursing Science Quarterly, 12, 164-169.

[25] Dickenset, C., Jackson, J., Tomenson, B., Hay, E. and Creed, F. (2004) Association of depression and rheumatoid arthritis. Psychosomatics, 44, 209-215. doi:10.1176/appi.psy.44.3.209

[26] Kiecolt-Glaser, J.K., McGuire, L., Robles, T.F. and Glaser, R. (2002) Psychoneuroimmunology: Psychological influences on immune function and health. Journal of Consulting and Clinical Psychology, 70, 537-547. doi:10.1037/0022-006X.70.3.537

[27] Hamilton, N., Zautra, A., Alex, J. and Reich, J.W. (2005) Affect and pain in rheumatoid arthritis: Do individual differences in affective regulation and affective intensity predict emotional recovery from pain? Annals of behavior 
Medicine, 29, 216-224. doi:10.1207/s15324796abm2903 8

[28] Hamilton, N.A., Karoly, P. and Kitzman, H. (2004) Selfregulation and chronic pain: The role of emotion. Cognitive Therapy and Research, 28, 559-576. doi:10.1023/B:COTR.0000045565.88145.76

[29] Christensen, A.J., Wiebe, J.S., Edwards, D.L., Micheles, J.D. and Lawton, W.J. (1996) Body consciousness, illness-related impairment, and patient adherence in hemodialysis. Journal of Consulting Clinical Psychology, 64, 147-152. doi:10.1037/0022-006X.64.1.147

[30] Arnett, F.C., Edworthy, S.M. and Bloch, D.A. (American Rheumatism Association) (1987) A revised criteria for classification of rheumatoid arthritis, from 1998. Arthritis \& Rheumatism, 31, 315-324. doi:10.1002/art.1780310302

[31] Sandqvist, G., Archenholtz, B., Scheja, A. and Hesselstrand, R. (2011) The Swedish version of the Multidimensional Assessment of Fatigue (MAF) in systemic sclerosis: Reproducibility and correlations to other fatigue instruments. Scandinavian Journal of Rheumatology, 40, 493-494. doi:10.3109/03009742.2011.605395

[32] Dixon, J.S. and Bird, H.A. (1981) Reproducibility along a $10 \mathrm{~cm}$ vertical visual analogue scale. Annals of the Rheumatic Diseases, 40, 87-89. doi:10.1136/ard.40.1.87

[33] Prevoo, M.L., van’t Hof, M.A., Kuper, H.H., van Leeuwen, M.A., van dePutte, L.B. and van Riel, P.L. (1995) Modified disease activity scores that include twentyeight-joint counts. Development and validation in a prospective longitudinal study of patients with rheumatoid arthritis. Arthritis \& Rheumatism, 38, 44-48. doi:10.1002/art.1780380107

[34] EuroQol Group (1990) EuroQol: A new facility for the measurement of health-related quality of life. Health Policy, 16, 199-208. doi:10.1016/0168-8510(90)90421-9

[35] Hurst, N.P., Kind, P., Ruta, D., Hunter, M., and Stubbings, A. (1997) Measuring health-related quality of life in rheumatoid arthritis: Validity, responsiveness and reliability of EuroQol (EQ-5D). British Journal of Rheumatology, 36, 551-559. doi:10.1093/rheumatology/36.5.551

[36] Baas, L.S., Berry, T.A., Allen, G., Wizer, M. and Wagoner, L.E. (2004) An exploratory study of body awareness in persons with heart failure treated medically or with transplantation. Journal of Cardiovascular Nursing, 19, 32-40.

[37] Lööf, H., Johansson, U.-B., Welin Henriksson, E., Lindblad, S. and Saboonchi, F. (2012) Development and psychometric testing of the Swedish version of the body awareness questionnaire. Journal of Advanced Nursing. doi:10.1111/jan.12020

[38] Gross, J.J. and John, O.P. (2003) Individual differences in two emotion regulation processes: Implications for affect, relationships, and well-being. Journal of Personality and Social Psychology, 85, 348-362. doi:10.1037/0022-3514.85.2.348

[39] Cohen, S., Kamarck, T. and Mermelstein, R. (1983) A global measure of perceived stress. Journal of Health Social Behavior, 24, 385-396. doi:10.2307/2136404
[40] Eskin, M. and Parr, D. (1996) Introducing a Swedish version of an instrument measuring mental stress. Reports from the Department of Psychology, Stockholm University, Stockholm.

[41] Watson, D., Clark, L.A. and Tellegen, A. (1988) Development and validation of brief measures of positive and negative affect: The PANAS scales. Journal of Personality and Social Psychology, 54, 1063-1070. doi:10.1037/0022-3514.54.6.1063

[42] Nahlén, C. and Saboonchi, F. (2010) Coping, sense of coherence and the dimensions of affect in patients with chronic heart failure. European Journal of Cardiovascular Nursing. 9, 118-125.

doi:10.1016/j.ejcnurse.2009.11.006

[43] Undén, A.L. and Orth-Gomer, K. (1989) Development of a social support instrument for use in population surveys. Social Science and Medicine, 29, 1387-1392. doi:10.1016/0277-9536(89)90240-2

[44] Davidson, R.J. (1993) The neuropsychology of emotion and affective style. In: M. Lewis and J. M. Haviland, Eds., Handbook of Emotion, Guilford Press, New York, 143154.

[45] Carver, C.S. and Scheier, M.F. (1990) Principles of selfregulation: Action and emotion. In: E. T. Higgins and R. M. Sorrentino, Eds., Handbook of Motivation and Cognition: Foundations of Social Behavior, The Guilford Press, New York, 3-52.

[46] Strand, V. and Khanna, D. (2010) The impact of rheumatoid arthritis and treatment on patients' lives. Clinical and Experimental Rheumatology, 28, 32-40.

[47] Harrison, M.J., Tricker, K.J., Davies, L., Hassell, A., Dawes, P., Scott, D.L., Knight, S., Davis, M., Mulherin, D. and Symmons, D.P.M. (2005) The relationship between social deprivation, disease outcome measures, and response to treatment in patients with stable, long-standing rheumatoid arthritis. Journal of Rheumatology, 32, 2330-2336.

[48] Eurenius, E., Brodin, N., Lindblad, S., Opava, C., and PARA Study Group (2007) Predicting physical activity and general health perception among patients with rheumatoid arthritis. Journal of Rheumatology, 34, 10-15.

[49] Engel, G.L. (1980) The clinical application of the biopsychosocial model. American Journal of Psychiatry, 137, 535-544.

[50] Frankel, R.M., Quill, T.E. and McDaniel, S.H. (2003) The biopsychosocial approach: Past, present, and future. University of Rochester Press, Rochester.

[51] Polit, D.F. and Beck, C.T. (2012) Nursing research: Generating and assessing evidence for nursing practice. 9th Edition, Wolters Kluwe Health/Lippincott Williams \& Wilkins, Philadelphia.

[52] Englund, M., Joud, A., Geborek, P., Felson, D.T., Jacobsson, L.T. and Petersson, I.F. (2010) Prevalence and incidence of rheumatoid arthritis in southern Sweden 2008 and their relation to prescribed biologics. Rheumatology, 49, 1563-1569. doi:10.1093/rheumatology/keq127 